\title{
MRI in early detection of prostate cancer
}

\author{
Francesco Giganti ${ }^{a, b}$, Caroline M Moore ${ }^{b, c}$
}

a. Department of Radiology, University College London Hospital NHS Foundation Trust, London, UK

b. Division of Surgery \& Interventional Science, University College London, London, UK

c. Department of Urology, University College London Hospital NHS

Foundation Trust, London, UK

Corresponding author:

Francesco Giganti, MD

Division of Surgery and Interventional Science,

University College London, 3rd Floor,

Charles Bell House, 43-45 Foley St.,

London, United Kingdom

W1W 7TS

email: f.giganti@ucl.ac.uk

phone number: +44 (0)20 35495778 


\section{$\underline{\text { Abstract }}$}

Purpose of review: The use of magnetic resonance imaging (MRI) in the early detection of prostate cancer (PCa) is increasing rapidly. In the last couple of years, there have been a number of key publications which have led to its adoption in the UK and European guidelines.

Recent findings: PROMIS showed that standard biopsy missed up to half of clinically significant disease, compared to $5 \mathrm{~mm}$ template mapping biopsy. Three studies then compared the standard transrectal ultrasound (TRUS) pathway to an $\mathrm{MRI}+/$ - targeted biopsy pathway. These showed that MRI-targeted biopsies detect more clinically significant disease and reduce overdetection of indolent disease whilst allowing between one third to one half of men to avoid an immediate biopsy. Cost effectiveness data show that using MRI to determine who gets a biopsy and how that biopsy is done is a cost-neutral approach when men at lowest risk do not undergo biopsy.

Summary: Prostate MRI is a useful and cost-effective tool for early detection of PCa which minimises the impact of overdetection and overtreatment whilst maximising the detection of PCa which could benefit from treatment. The next challenge is to ensure that centres offering MRI are able to offer high quality MRI acquisition and reporting.

Keywords: MRI, prostate cancer, early detection, risk prediction 


\section{Key points:}

- Prostate MRI and targeted biopsies are more accurate at detecting clinically significant $\mathrm{PCa}$, reduce overdiagnosis of indolent disease by $50 \%$ and allow between one third and one half of men to safely avoid immediate biopsy.

- Prostate MRI is a useful and cost-effective tool for early detection of PCa.

- A number of national and international guidelines have formally recommended MRI before prostate biopsy. 


\section{Introduction}

The traditional pathway for prostate cancer (PCa) diagnosis uses systematic random transrectal ultrasound (TRUS) biopsy in response to an elevated prostate specific antigen (PSA) and/or abnormal digital rectal examination (DRE). Recent data has shown that, when compared to a $5 \mathrm{~mm}$ mapping prostate biopsy, this approach misses half of clinically significant PCa in men having a first biopsy [1**]. Further work comparing a standard biopsy pathway to an MRI-led pathway has firmly established that MRI before biopsy allows greater detection of clinically significant disease, reduces the overdiagnosis of indolent disease and allows between one third and one half of men to safely avoid an immediate biopsy. $\left[2^{*}, 3^{\star *}, 4^{\star \star}\right]$

There has been a rapid evolution of multiparametric magnetic resonance imaging (mpMRI) of the prostate over the last couple of decades $\left[5^{\star}\right]$ using a combination of different MR sequences, such as T2-weighted (T2-WI), diffusion-weighted (DWI) and dynamic contrast- enhancement (DCE) imaging. T2-WI is the most useful technique to study the anatomy of the gland, while DWI assesses the free movement of water molecules within tissues, which is restricted in PCa. Rapid acquisition of images after contrast administration (DCE) allows the leaky and disorganised vasculature of new tumours to show characteristic rapid uptake and rapid washout.

We discuss here the most recent publications in the field and their impact on national and international guidelines. 


\section{MRI as the first assessment of men with a raised PSA or abnormal DRE}

The important questions asked of MRI in this setting are - how does an MRI-led pathway compare to a standard biopsy pathway? If the MRI is negative, can a biopsy be safely avoided? Questions also arise about which other factors (e.g. PSA density, PSA kinetics, family history) should be taken into account when considering biopsy in men with no definitive lesion on MRI.

\section{How does an MRI-led pathway compare to a standard biopsy pathway?}

The PROstate Mri Imaging Study (PROMIS) study [1**] compared mpMRI and standard TRUS biopsies in biopsy-naïve men using $5 \mathrm{~mm}$ template mapping prostate biopsies as a reference test. Using a definition of significance of any Gleason primary pattern 4 , or $6 \mathrm{~mm}$ of any cancer, TRUS biopsy detects around half of the clinically significant cancer that is found at $5 \mathrm{~mm}$ template mapping biopsy, whilst MRI detects $93 \%$ of these cancers. Around 1 in 4 men could safely avoid a biopsy when the MRI is deemed low risk.

It is important to note that MRI-targeted biopsies were not performed in PROMIS $\left[1^{* *}\right]$, and comparison is between MRI and TRUS biopsy, with $5 \mathrm{~mm}$ sampling as the reference standard. The performance characteristics of both MRI and standard TRUS biopsy vary with the definition of clinically significant cancer used. Critics point out that when using Gleason $3+4$ as the definition of clinically significant cancer the negative predictive value of MRI was $76 \%$ suggesting that one in 4 clinically significant cancers could be missed on MRI. Others would argue that not all Gleason 
$3+4$ needs to be detected, and that this is not a reason to negate the role of $M R I$ in the decision to undertake biopsy or not. However, it should also be remembered that no-one is suggesting the routine use of the 'gold standard' diagnostic test of $5 \mathrm{~mm}$ template mapping biopsy, due to its significant cost and morbidity implications. Despite the fact that men with a prostate volume of $>100 \mathrm{mls}$ were excluded, the rate of urinary retention was $10 \%$ (58/576) in PROMIS. In addition, a significant number of men had new onset problems with erections $(76 / 576,14 \%)$.

These side effects of an intensive mapping biopsy were also seen in the PICTURE study [6]. It assessed men who had had a previous TRUS biopsy, and used a combination of a $5 \mathrm{~mm}$ transperineal mapping biopsy, and biopsies targeted to MRI lesions. Acute urinary retention was seen in $23 \%$ (56/249) and erectile dysfunction at 30 days in $20 \%$ of men (49/249).

PROMIS established that MRI can more accurately detect clinically significant cancer than standard TRUS biopsy. The next question is whether these detection rates can be realised in clinical practice, as part of a diagnostic pathway. The potential for error here also includes the ability to target a lesion, which was not assessed in PROMIS. Three studies have been published in the last couple of years which directly address this question (Table 1).

The first is the PRECISION study, a multicentre randomised controlled trial where biopsy-naïve men either had a standard or an MRI-targeted biopsy, published in March 2018. [2*] Of men in the standard biopsy arm, 26\% (64/248) had clinically significant PCa (Gleason score $\geq 7$ ) whilst 22\% (55/248) had clinically insignificant 
cancer and could be considered 'overdiagnosed'. In the MRI arm $38 \%$ of men (95/252) had clinically significant cancer and 9\% (23/252) had clinically insignificant $\mathrm{PCa}$, and $28 \%(71 / 252)$ of men avoided biopsy.

The second study (called $4 M$ ), published in late $2018\left[3^{* *}\right]$ is a prospective multicentre Dutch study that included 626 biopsy-naïve men who underwent prebiopsy MRI followed by systematic TRUS-biopsy (TRUS pathway) and in-bore targeted biopsy (MRI pathway) in the case of suspicious mpMRI $(n=317)$. This study showed that the detection rate of clinically significant PCa (Gleason score $\geq 7$ ) is similar for the MRI and TRUS pathways (25\% and $23 \%$, respectively), with fewer insignificant PCa cases in the MRI pathway (25\% and 14\%, respectively). This study showed the highest rate of men who could avoid biopsy at $49 \%$.

The third study (MRI-FIRST) [4**, also published in late 2018 , is a prospective, multicentre French study of 251 men that compared the two biopsy techniques (systematic vs targeted) in each man. They found that $14 \%$ of men were able to avoid a biopsy, and that detection rates of clinically significant cancer (defined as Gleason $3+4$ disease) were similar with each technique (29.9\% with systematic biopsy and $32.3 \%$ with targeted biopsy; $p=0.38$ ).

The differences between PRECISION, 4M and MRI-first are likely to be related to the technique of both MRI acquisition and reporting, and of targeting technique, and possibly of underlying difference in risk between the study populations. The $4 \mathrm{M}$ study had the highest rate of allowing men to safely avoid biopsy, and this is likely to be due to fact that the MRIs were all done at $3 \mathrm{~T}$ and reviewed centrally by one of two 
radiologists before a biopsy decision was made. PRECISION was the study with the largest difference between the standard and MRI-targeted arms for detection of clinically significant cancer and the greatest number of targeted cores per lesion and per man (a maximum of 4 cores per lesion, and up to 12 targeted cores per man in the MRI arm).

\section{What is the likelihood of clinically significant cancer in a man with a negative or equivocal MRI?}

There is controversy over whether it is acceptable to omit or defer biopsy in men with a negative or equivocal MRI. The UK NICE guidelines [7] include the recommendation :

Consider omitting a prostate biopsy for people whose multiparametric MRI Likert score is 1 or 2, but only after discussing the risks and benefits with the person and reaching a shared decision

This is based on data showing that, in men with a negative MRI (scoring 1 or 2 on Likert or Prostate Imaging Reporting and Data System - PI-RADS - scores) the risk of diagnosing indolent disease if standard biopsy is done is far greater (up to 1 in 4 men) than the risk of missing clinically significant disease which could be detected by standard biopsy (around 2-3\%, depending on centre experience). As well as the published data available, it is important that each centre should be aware of their own data, especially the negative predictive value of MRI in their own pathway, 
before omitting standard biopsy. Gaziev and colleagues [8] showed a learning curve with negative predictive value increasing from $67 \%$ to $89 \%$ over time.

It is often urologists who carry out MRI-targeted biopsies either using visual or software assisted registration and improving the skills of urologists in reading MRI in order for them to carry out prostate biopsy or other procedures is important. There are a number of MRI courses designed for urologists. Kasivisvanathan and colleagues [9] ran a 2-day course which showed an improvement in the detection of clinically significant cancer with an improvement in the ROC curve from 0.60 to 0.77 .

Panebianco and colleagues [10**] have recently reported on 1255 men (659 biopsynaïve and 596 with a previously negative systematic TRUS biopsy) with 48-months biopsy and MRI follow-up after negative prostate MRI. The main finding of this study is that $95 \%$ of men in the first group and $96 \%$ of men in the second group were free from clinically significant disease after 48 months of follow up. Another group have recently reported that men with $\mathrm{PI}-\mathrm{RADS} \leq 2$ had $99.6 \%$ freedom from clinically significant disease at 3 years [11].

\section{Should standard cores be added to targeted cores?}

There is still much debate about whether standard cores should be added to MRItargeted cores. A recent review by Stabile and colleagues [12] looked at this and concluded that there was a statistically non-significant increase in the detection of clinically significant PCa (34\% to 39\%) when adding standard sampling to MRI- 
targeted biopsies, but that this was accompanied by an increase in the detection of indolent disease from $11 \%$ to $23 \%$.

\section{Inclusion of pre-biopsy MRI in recent guidelines}

In the last two years, the National Health Service (NHS) England [13], the UK National Institute for Health and Care Excellence (NICE) [7] and the European Association of Urology (EAU) [14] guidelines have recommended MRI before biopsy for all men fit for radical treatment.

\section{Management of indeterminate lesions on MRI: PI-RADS 3 and Likert 3}

An important aspect of mpMRI in the early detection of $\mathrm{PCa}$ is the management of indeterminate lesions (i.e. PI-RADS or Likert 3, where the presence of clinically significant PCa is equivocal) on mpMRI. First of all, it should be noted that the prevalence of such lesions is significant (around $30 \%$ of men) and varies between different cohorts (biopsy naïve, previously negative biopsies or active surveillance). [15]

Schoots and colleagues [15] report that there is still no robust evidence at present supporting a threshold of PI-RADS $\geq 4$ instead of PI-RADS $\geq 3$ to select men for MRItargeted biopsies. The management of MRI-indeterminate lesions in PCa has been

recently reviewed by Gomez-Rivas and colleagues. [16] Here the authors report that there is still no universally accepted definition of 'indeterminate lesions' on prostate MRI and that the strategies for their management (i.e. biopsy vs surveillance) should 
take into account different factors: i) different definitions of clinically significant PCa; ii) different mpMRI protocols; iii) different expertise in reporting. The authors concluded that the use of pre-biopsy MRI together with other clinical parameters, biomarkers and nomograms may allow more accurate risk stratification and assessment of the need for prostate biopsy.

There are also two papers from the United Kingdom that address the management of indeterminate lesion using a Likert scale, which places greater emphasis on radiologist interpretation than the more rigid PI-RADS system. Both a PI-RADS v 2.1 score of 3 and a Likert 3 are considered equivocal or indeterminate for clinically significant cancer. [17]

The first study by Van Der Sar and colleagues [18] described the outcomes of 168 men with a Likert 3 lesion who were offered one of two initial management strategies. The first strategy $(n=73)$ was an immediate targeted biopsy of the MRI lesion whilst the second strategy $(n=95)$ was a surveillance process including PSA monitoring and/or mpMRI at intervals of $6-12$ months, with biopsy on a for-cause basis. The overall proportion of men with clinically significant PCa detected was $14 \%$ (23/168) with no difference between the two groups.

The authors of the second paper [19] aimed to determine whether Likert $3 \mathrm{MR}$ studies could be categorised into significant/insignificant PCa by different parameters such as PSA, PSA density, rescoring according to PI-RADS v. 2 guidelines [20] and morphological MRI features. They retrospectively selected 330 men who had had a 3T mpMRI followed by 20-zone transperineal +/- targeted biopsy. Two readers 
independently assessed both scoring systems and classified MRI morphological features in consensus (clinically significant cancer was defined as Gleason $\geq 7$ ). PSA density was statistically different $(p=0.004)$ between significant and nonsignificant/no cancer groups and subjective Likert-assessment discriminated men with significant cancer better than PI-RADS v2. The inter-reader agreement was $83 \%$ for Likert and $56 \%$ for PI-RADS v2.

\section{Is PSA density helpful to decide who should get a biopsy?}

In case of a negative MRI with a high PSA density $(\geq 0.15 \mathrm{ng} / \mathrm{mL} / \mathrm{mL})$, Panebianco and colleagues $\left[10^{* *}\right]$ reported that PSA density had a hazard ratio of 7.57 for the diagnosis of clinically significant PCa, making a strong case that men with a high PSA density and negative MRI should be offered a biopsy.

A recent UK consensus meeting [21] recommends a set of criteria that are required for the practical dissemination of consistently high-quality prostate $\mathrm{mpMRI}$ as a diagnostic test before biopsy in men at risk for PCa. It is recommended to use MRIbased volumes to assess PSA density to aid decision making in men with equivocal MRI scores [19, 22, 23]. At present, a threshold of $0.15 \mathrm{ng} / \mathrm{mL} / \mathrm{mL}$ is suggested in the diagnostic setting $[22,23]$ although some centres may adopt a more conservative approach, using a lower threshold (e.g. $0.12 \mathrm{ng} / \mathrm{mL} / \mathrm{mL}$ ) but including other risk factors in their decision-making process.

Is MRI cost-effective in the early detection of prostate cancer? 
Cost-effectiveness data from PROMIS [24] revealed that the MRI pathway is effective for the diagnosis of clinically significant $\mathrm{PCa}$, as the use of an upfront MR scan with up to two MRI-targeted TRUS biopsies detects more clinically significant PCa per pound spent than a strategy using TRUS biopsy first (sensitivity $=0.95$ vs 0.91 ) and is cost effective (incremental cost-effectiveness ratio of $£ 7,076$ [€8350/quality-adjusted life years gained]).

Barnett and colleagues [25] report on the application of a dedicated Markov model to estimate the cost-effectiveness of MRI and targeted fusion biopsy for the early detection of $\mathrm{PCa}$ in men with elevated PSA ( $4 \mathrm{ng} / \mathrm{mL}$ ) receiving $\mathrm{mpMRI}$ followed by targeted (or combined, targeted + standard) biopsy on positive MRI, and standard or no biopsy on negative MRI (PI-RADS scoring system). A combined biopsy (targeted + standard) in PI-RADS $\geq 3$ and no biopsy in PI-RADS $<3$ were the optimum screening strategy. The authors concluded that the MRI pathway is cost-effective in the early detection of PCa.

\section{What is the inter-observer variability of prostate MRI?}

Despite efforts to improve MRI reporting such as the introduction of PI-RADS v2.1 guidelines [17], inter-observer variability remains problematic for prostate MRI, especially for less experienced radiologists.

Greer and colleagues [26] have recently evaluated the agreement among nine radiologists with difference levels of experience (high, intermediate, and low) in detecting and assessing PCa at MRI using PI-RADS v.2.0 guidelines [20]. The 
interobserver agreement was excellent for detecting the index lesions $(k=0.87)$ but the agreement on PI-RADS v.2.0 category assignment of index lesions was moderate $(\mathrm{k}=0.42)$. Specifically, the proportion of agreement for PI-RADS 4 lesions in the transition zone was 0.25 for T2-WI, 0.82 on DCE and 0.59 on DWI.

Another recent study by Pickersgill and colleagues [27] determined the variability in prostate MRI reporting according to PI-RADS v.2.0 guidelines [20] among four radiologists with different levels of expertise. The authors found significant differences using a threshold of PI-RADS $>3 /$ Gleason $>7$ and PI-RADS $>4 /$ Gleason $>6$ as cut-offs ( $p<0.01$ and $p=0.02$, respectively).

It is clear that the PI-RADS v.2.0 guidelines [20] generate substantial difference in the reproducibility of MR features among radiologists. Some of the ambiguities and limitations of PI-RADS v. 2.0 have been documented and addressed in PI-RADS v. 2.1 [17]. Such amendments will hopefully improve inter-reader variability and simplify the interpretation system further, but additional studies and investigative data are needed.

There is hope that computer aided detection (CAD) might be of use in addressing this, with a recent study suggesting that CAD maps for the radiologist to review led to increased detection of larger lesions. [28] It should also be recognised that differences in MRI acquisition, as well as inter-reader variability can play a large role in variation in reports across different institutions. [29]

\section{Conclusion}


There is compelling evidence to support the use of mpMRI in the early diagnosis of PCa. In some countries prebiopsy MRI is already well established and the robust data published over the last two years encourage the incorporation of $\mathrm{mpMRI}$ as a standard part of the assessment for all men at risk of PCa. Men with equivocal or negative mpMRI should have a risk assessment including PSA density, and a personalised discussion of the risks and benefits on an individual basis.

Acknowledgements: none

Financial support and sponsorship: Francesco Giganti is funded by the UCL Graduate Research Scholarship and the Brahm PhD scholarship in memory of Chris Adams.

Conflicts of interest: none. 


\section{References}

1. ${ }^{* \star}$ Ahmed HU, Bosaily AE, Brown LC, et al. Diagnostic accuracy of multiparametric MRI and TRUS biopsy in prostate cancer (PROMIS): a paired validating confirmatory study. Lancet $2017 ; 6736: 1-8$.

In this study the most cost-effective strategy involved testing all men with prostate $M R I$, followed by an MRI-directed biopsy in those with suspected clinically significant prostate cancer (MRI pathway), followed by re-biopsy if clinically significant prostate cancer was not detected. This strategy was cost-effective and detected 95\% of clinically significant prostate cancer.

2. * Kasivisvanathan V, Rannikko AS, Borghi M, et al. MRI-Targeted or Standard

Biopsy for Prostate-Cancer Diagnosis. N Engl J Med 2018; 378:1767-1777.

In the PRECISION trial the MRI pathway avoided $28 \%$ of biopsy procedures. The use of risk assessment with MRI before biopsy and MRI-targeted biopsy was superior to standard transrectal ultrasonography-guided biopsy in biopsy-naïve men at clinical risk for prostate cancer.

3. ${ }^{* *}$ van der Leest M, Cornel E, Israël B, et al. Head-to-head Comparison of

Transrectal Ultrasound-guided Prostate Biopsy Versus Multiparametric

Prostate Resonance Imaging with Subsequent Magnetic Resonance-guided

Biopsy in Biopsy-naïve Men with Elevated Prostate-specific Antigen: A Large

Prospective Mu. Eur Urol 2019; 75(4):570-578.

In the $4 M$ trial the $M R I$ pathway detected $25.4 \%$ men with csPCa vs $23.3 \%$ by systematic biopsy. The MRI pathway detected $14.1 \%$ men with Grade 1 vs $24.8 \%$ by systematic biopsy. The MRI pathway detected an equivalent proportion of men with csPCa (absolute difference 2.1\%) and beneficially reduced the detection of Grade 1 (absolute difference 10.7\%).

4. $\quad{ }^{* *}$ Rouvière $\mathrm{O}$, Puech $\mathrm{P}$, Renard-Penna $\mathrm{R}$, et al. Use of prostate systematic and targeted biopsy on the basis of multiparametric MRI in biopsy-naive patients (MRI-FIRST): a prospective, multicentre, paired diagnostic study. Lancet Oncol 2019; 20(1):100-109. 
In the MRI-first study the MRI pathway detected $32.3 \%$ men with csPCa vs $29.9 \%$ by systematic biopsy. The MRI pathway detected 9.2\% men with Grade 1 vs $22.3 \%$ (56/251) by systematic biopsy. The MRI pathway detected an equivalent proportion of cSPCa (absolute difference 2.4) and beneficially reduced the detection of Grade 1 PCa (absolute difference 13.1\%). Obtaining a multiparametric MRI before biopsy in biopsy-naive men can improve the detection of clinically significant prostate cancer but does not seem to avoid the need for systematic biopsy.

5. * Giganti F, Rosenkrantz AB, Villeirs GM, et al. The evolution of MRI of the prostate: the Past, the Present, and the Future. AJR Am J Roentgenol 2019; 30:1132019.

In this review, the authors discuss the evolution of MRI in prostate cancer from the early 1980s to the current day, providing analysis of the key studies on this topic.

6. Miah S, Eldred-Evans D, Simmons LAM, et al. Patient Reported Outcome Measures for Transperineal Template Prostate Mapping Biopsies in the PICTURE Study. J Urol 2018; 200:1235-1240

7. https://www.nice.org.uk/guidance/NG131 (2019). Accessed $3^{\text {th }}$ July 2019.

8. Gaziev G, Wadhwa K, Barrett T, et al. Defining the learning curve for multiparametric magnetic resonance imaging (MRI) of the prostate using MRItransrectal ultrasonography (TRUS) fusion-guided transperineal prostate biopsies as a validation tool. BJU Int 2016; 117:80-86 . doi: 10.1111/bju.12892

9. Kasivisvanathan V, Ambrosi A, Giganti F, et al. A Dedicated Prostate MRI Teaching Course Improves the Ability of the Urologist to Interpret Clinically Significant Prostate Cancer on Multiparametric MRI. Eur Urol 2019; 75(1):203204.

10. ** Panebianco V, Barchetti G, Simone G, et al. Negative Multiparametric Magnetic Resonance Imaging for Prostate Cancer: What's Next?. Eur Urol 2018; 74:48-54.

In this study the authors report 1255 men with 48-months biopsy and MRI follow-up 
after negative prostate MRI. In this cohort, there were 659 biopsy-naïve men with a negative MR scan (group A), and 596 with prior negative biopsy (group B). Anygrade $P C a$ and csPCa diagnosis-free survival probabilities after two years of followup were $94 \%$ and $95 \%$, respectively, in group $A$ and $96 \%$ in group $B$.

11. Venderink W, van Luijtelaar A, van der Leest M, et al. Multiparametric MRI and follow-up to avoid prostate biopsy in 4259 men. BJU Int 2019 (in press). doi: 10.1111/bju.14853

12. Stabile A, Giganti F, Emberton M, Moore CM. MRI in prostate cancer diagnosis: do we need to add standard sampling? A review of the last 5 years. Prostate Cancer Prostatic Dis 2018;21(4):473-487.

13. NHS England Recommendations. https://www.england.nhs.uk/wpcontent/uploads/2018/04/implementing-timed-prostate-cancer-diagnosticpathway.pdf. Accessed $3^{\text {th }}$ July 2019.

14. European Association of Urology. EAU guidelines on prostate cancer. EAU https://uroweb.org/guideline/prostate-cancer/ (2019). Accessed $3^{\text {th }}$ July 2019.

15. Schoots IG. MRI in early prostate cancer detection: how to manage indeterminate or equivocal PI-RADS 3 lesions? Transl Androl Urol 2018; 7:7082.

16. Rivas JG, Giganti F, Álvarez-Maestro M, et al.Prostate Indeterminate Lesions on Magnetic Resonance Imaging-Biopsy Versus Surveillance: A Literature Review. Eur Urol Focus 2018; (in press) doi: 10.1016/j.euf.2018.02.012

17. Turkbey B, Rosenkrantz AB, Haider MA et al. Prostate Imaging-Reporting and Data System Version 2.1: 2019 Update of Prostate Imaging Reporting and Data System Version 2. Eur Urol 2019; (in press) doi:

10.1016/j.eururo.2019.02.033

18. van der Sar ECA, Kasivisvanathan V, Brizmohun M, et al. Management of Radiologically Indeterminate Magnetic Resonance Imaging Signals in Men at 
Risk of Prostate Cancer. Eur Urol Focus 2019; 5(1):62-68.

19. Appayya MB, Sidhu HS, Dikaios N, et al. Characterizing indeterminate (Likertscore 3/5) peripheral zone prostate lesions with PSA density, PI-RADS scoring and qualitative descriptors on multiparametric MRI. BJ Radiol 2018; 91(1083):20170645.

20. Weinreb JC, Barentsz JO, Choyke PL, et al. PI-RADS Prostate Imaging Reporting and Data System: 2015, Version 2. Eur Urol (2016); 69(1):16-40.

21. Brizmohun Appayya M, Adshead J, Ahmed H, et al. National Implementation of multi-parametric MRI for prostate cancer detection - recommendations from a UK consensus meeting. BJU Int 2018; 122(1):13-25

22. Washino S, Okochi T, Saito K, et al. Combination of prostate imaging reporting and data system (PI-RADS) score and prostate-specific antigen (PSA) density predicts biopsy outcome in prostate biopsy naïve patients. BJU Int 2017: 119:225-233.

23. Distler FA, Radtke JP, Bonekamp D, et al. The Value of PSA Density in Combination with PI-RADS ${ }^{T M}$ for the Accuracy of Prostate Cancer Prediction. J Urol 2017: 198:575-582

24. Faria R, Soares MO, Spackman E, et al. Optimising the Diagnosis of Prostate Cancer in the Era of Multiparametric Magnetic Resonance Imaging: A Costeffectiveness Analysis Based on the Prostate MR Imaging Study (PROMIS). Eur Urol 2018;73:23-30

25. Barnett CL, Davenport MS, Montgomery JS, et al. Cost-effectiveness of magnetic resonance imaging and targeted fusion biopsy for early detection of prostate cancer. BJU Int 2018; 122:50-58.

26. Greer MD, Shih JH, Lay N, et al. Interreader Variability of Prostate Imaging 
Reporting and Data System Version 2 in Detecting and Assessing Prostate Cancer Lesions at Prostate MRI. AJR Am J Roentgenol 2019; (in press) doi: 10.2214/AJR.18.20536

27. Pickersgill NA, Vetter JM, Andriole GL, et al. Accuracy and Variability of Prostate Multiparametric Magnetic Resonance Imaging Interpretation Using the Prostate Imaging Reporting and Data System: A Blinded Comparison of Radiologists. Eur Urol Focus 2018; (in press) doi: 10.1016/j.euf.2018.10.008

28. Giannini V, Mazzetti S, Armando E, et al. Multiparametric magnetic resonance imaging of the prostate with computer-aided detection: experienced observer performance study. Eur Radiol 2017; 10:4200-4208

29 Müller S, Lilleaasen G, Sand TE, et al. Poor reproducibility of PIRADS score in two multiparametric MRIs before biopsy in men with elevated PSA. World $\mathrm{J}$ Urol. 2018;36(5):687-691. 